\title{
The Renilla luciferase gene as a reference gene for normalization of gene expression in transiently transfected cells
}

Meesbah Jiwaji ${ }^{1}$, Rónán Daly², Kshama Pansare ${ }^{1}$, Pauline McLean ${ }^{1}$, Jingli Yang ${ }^{1}$, Walter Kolch³ ${ }^{3}$ Andrew R Pitt ${ }^{*}$

\begin{abstract}
Background: The importance of appropriate normalization controls in quantitative real-time polymerase chain reaction (qPCR) experiments has become more apparent as the number of biological studies using this methodology has increased. In developing a system to study gene expression from transiently transfected plasmids, it became clear that normalization using chromosomally encoded genes is not ideal, at it does not take into account the transfection efficiency and the significantly lower expression levels of the plasmids. We have developed and validated a normalization method for qPCR using a co-transfected plasmid.

Results: The best chromosomal gene for normalization in the presence of the transcriptional activators used in this study, cadmium, dexamethasone, forskolin and phorbol-12-myristate 13-acetate was first identified. qPCR data was analyzed using geNorm, Normfinder and BestKeeper. Each software application was found to rank the normalization controls differently with no clear correlation. Including a co-transfected plasmid encoding the Renilla luciferase gene (R/uc) in this analysis showed that its calculated stability was not as good as the optimised chromosomal genes, most likely as a result of the lower expression levels and transfection variability. Finally, we validated these analyses by testing two chromosomal genes (B2M and ActB) and a co-transfected gene (Rluc) under biological conditions. When analyzing co-transfected plasmids, Rluc normalization gave the smallest errors compared to the chromosomal reference genes.

Conclusions: Our data demonstrates that transfected Rluc is the most appropriate normalization reference gene for transient transfection qPCR analysis; it significantly reduces the standard deviation within biological experiments as it takes into account the transfection efficiencies and has easily controllable expression levels. This improves reproducibility, data validity and most importantly, enables accurate interpretation of qPCR data.
\end{abstract}

\section{Background}

The analysis of gene expression using quantitative realtime polymerase chain reaction (qPCR) has become increasingly important as biological research has focused on developing insights into the complex regulatory networks that exist within cells [1]. qPCR is often the assay of choice as it is sensitive and reproducible; it allows the simultaneous analysis of gene expression in a number of different samples and as a result of the high dynamic range, this technique is suitable even when only a few cells are available. The speed of analysis and

\footnotetext{
* Correspondence: a.pitt@bio.gla.ac.uk

${ }^{1}$ Institute of Molecular, Cell and Systems Biology, College of Medical, Veterinary and Life Sciences, University of Glasgow, Glasgow, G12 8QQ, UK Full list of author information is available at the end of the article
}

the potential for automation and multiplexing makes qPCR an attractive technique for the analysis of gene expression [2-4].

Unfortunately, problems attributed to the biological and technical variability which can occur between the different steps of the experimental procedures, are associated with the qPCR assay. The technical variables include the amount of starting materials in the reactions, the quality of the RNA samples and the efficiency of the enzymatic steps (i.e. reverse transcription and PCR) $[5,6]$. The biological variables include the differences in the levels of transcriptional expression of genes between tissues and cell types [7]. To take into account these variations, internal reference genes are often used to normalize the qPCR data $[8,9]$. Ideally, the internal
C Biomed Central

() 2010 Jiwaji et al; licensee BioMed Central Ltd. This is an Open Access article distributed under the terms of the Creative Commons Attribution License (http://creativecommons.org/licenses/by/2.0), which permits unrestricted use, distribution, and reproduction in any medium, provided the original work is properly cited. 
reference gene should be expressed at levels comparable to the gene of interest and the levels of expression of the gene selected as the internal reference should not vary between the samples and treatments selected for analysis $[8,10]$. The selection of the most appropriate internal reference gene serves to decrease the error both within the experiment and between biological experiments $[8,9,11]$. In addition, it allows valid analyses of qPCR data to be conducted [10]. This makes the selection of the internal reference an important factor in the design of a qPCR experiment. This becomes particularly important when the sequence targeted for analysis is being transiently transfected into cells; however validated methods for this type of experiment are not currently available.

In a living cell, it is unlikely that the transcription of any gene is resistant to changes in the cell cycle or in the levels of nutrients. It is therefore important that in the selection of the reference gene, the candidate genes should be regulated at a minimal level. A number of studies have shown that the classical internal reference genes, such as the glyceraldehyde-3-phosphate dehydrogenase gene (GAPDH), are not always the most suitable reference genes [12], and that the levels of GAPDH mRNA fluctuate in the cell. This is understandable considering the many pathways in which this protein is involved, including endocytosis, translational control, export of nuclear tRNA, DNA replication and repair, apoptosis and glycolysis [12]. GAPDH was originally selected as a normalization reference as the gene encoded a protein with a 'housekeeping' function. It was not until later that the role of GAPDH in the cell was more fully understood, and thus its potential unsuitability as an internal reference. It is, therefore, important that the design of a biological study includes the evaluation of potential internal reference genes, and that the most appropriate reference genes are selected. The availability of software applications such as geNorm, Normfinder and BestKeeper, that use statistical methods to select the most appropriate internal reference genes, make this task easier [13-15].

Most of the traditional internal reference genes are chromosomal genes. The use of a chromosomal internal reference takes into account all the technical and biological variables that are present within the experiment bar one. None of the internal reference genes, as long as the gene was present on the chromosomal DNA, would take into account the variation in the transfection efficiency between samples that had been transiently transfected with plasmid DNA encoding a gene of interest. Also, choosing a genomically encoded gene with an appropriate expression level is challenging. We have adapted a technique often applied to the normalization of reporter enzyme activity to $\mathrm{qPCR}$, and have demonstrated that using a co-transfected plasmid-encoded Renilla reniformis luciferase gene $(R l u c)$ as an internal reference is more appropriate for the normalization of qPCR data in transiently transfected systems.

\section{Results and Discussion}

We are developing a plasmid vector to study transcriptional activation derived from cis-acting regulatory elements of interest in transiently transfected mammalian cell lines. We planned to validate the putative cis-acting regulatory elements using a reporter enzyme system that could be adapted to allow a high-throughput analysis of the samples of interest. Traditional reporter enzyme assays usually use the activity of one reporter enzyme to normalize the activity of another enzyme of interest when two plasmids, each separately encoding one of the enzymes, have been transiently transfected into cells. One of the most common systems is the firefly luciferase-Renilla luciferase enzyme system, where the enzyme activity of Renilla luciferase (encoded by the control plasmid) is used to normalize the firefly luciferase enzyme activity (present on the experimental plasmid).

A complete workflow for the firefly luciferase-Renilla luciferase system from the plasmid constructs used to transfect the cells to the reporter enzyme assay system used to quantify the reporter enzyme activity has been commercially developed (Promega). This uses one of three control plasmid constructs with different promoters upstream of the Rluc gene [16]. pRL-SV40, which encodes the early simian virus 40 (SV40) enhancer/promoter region, and pRL-CMV, which encodes the cytomegalovirus (CMV) immediate early enhancer/promoter region both provide high levels of Renilla luciferase enzyme expression. In contrast, pRL-TK encodes the weaker herpes simplex virus (HSV) thymidine kinase (TK) promoter, resulting in lower levels of Renilla luciferase enzyme expression.

For the studies described here the most appropriate system would have a promoter upstream of Renilla luciferase with relatively high expression levels such that a low concentration of the control plasmid could be used in the transient transfections. It would also be advantageous to use a promoter that did not encode transcription factor binding sequences. All three control plasmids provided as part of the commercial kit have their limitations. Breuning et al. [17] and Svensson et al. [18] reported that the CMV promoter was susceptible to up-regulation in mammalian cells under conditions of cell stress. This is not ideal since in cis-regulatory element studies it is likely that some of the treatments used to activate the cis-regulatory elements will result in cell stress. A number of reports have also highlighted that HSV TK promoter is susceptible to altered regulation under a range of experimental conditions [19-21]. 
Ho et al. [21] performed a comparison between pRL-TK and pRL-SV40 and stated that of the two plasmids, pRL-SV40 was less susceptible to non-specific activation by the GATA transcription factor than pRL-TK. As this project aims to study cis-acting regulatory elements, we wanted to select the control plasmid that was least affected by undesired transcriptional regulation so we selected pRL-SV40 as the control plasmid.

To test pRL-SV40 (the control plasmid encoding Rluc), human embryonic kidney (HEK) 293 cells were transiently tranfected with an increasing concentration of pRL-SV40. Using a qPCR assay, expression of Rluc was normalized to the expression of $A c t B$, a chromosomal gene that is often used as a reference control [12]. As the DNA concentration of pRL-SV40 used to transfect the cells increased, increasing Rluc expression was detected (Figure 1) and optimal Rluc expression was detected when HEK293 cells were transfected with $100 \mathrm{ng}$ of control plasmid DNA under the conditions being used. In these experiments, we needed to balance the concentration of the control plasmid used in the transfections with the level of Rluc expression. The lowest concentration of control plasmid DNA at which consistent levels of Rluc gene expression were obtained was with $50 \mathrm{ng}$ of pRL-SV0 so this concentration of control plasmid DNA was used in subsequent experiments. It is interesting to note that the use of a transfected gene for normalization is advantageous in that the levels of the transfected gene can easily be adjusted to be suitable for a broad range of target genes by altering the absolute amount of plasmid DNA in the transfection.

HEK293 cells were transiently tranfected with pRLSV40 (50 ng) and an equivalent concentration of our reporter system plasmids pMN85 or pMN86. The concentration of pMN85/pMN86 was determined in an experiment similar to the one that was conducted for pRL-SV40 (data not shown). The control plasmid, pRLSV40, is $3.7 \mathrm{~kb}$ in size compared to pMN85/pMN86, both of which are $5.1 \mathrm{~kb}$ in size. Yin et al. [22] reported that there was an inverse correlation between the plasmid size and the transfection efficiency of that plasmid. As both the reporter system plasmids are of comparable size (pMN86 is 82 bp larger than pMN85), we determined that the transfection efficiencies between these plasmids would not be significantly different. In addition, despite the differences in the transfection efficiencies of the reporter system plasmids and the control plasmid, the transfection efficiencies of the respective plasmids should be comparable between samples allowing us to normalize the data derived from the reporter system plasmids to that of pRL-SV40, the control plasmid.

Plasmid pMN85 is the control vector which encodes the thymidine kinase promoter $\left(\mathrm{P}_{\mathrm{TK}}\right)$ upstream of the firefly (Photinus pyralis) luciferase gene (Fluc).

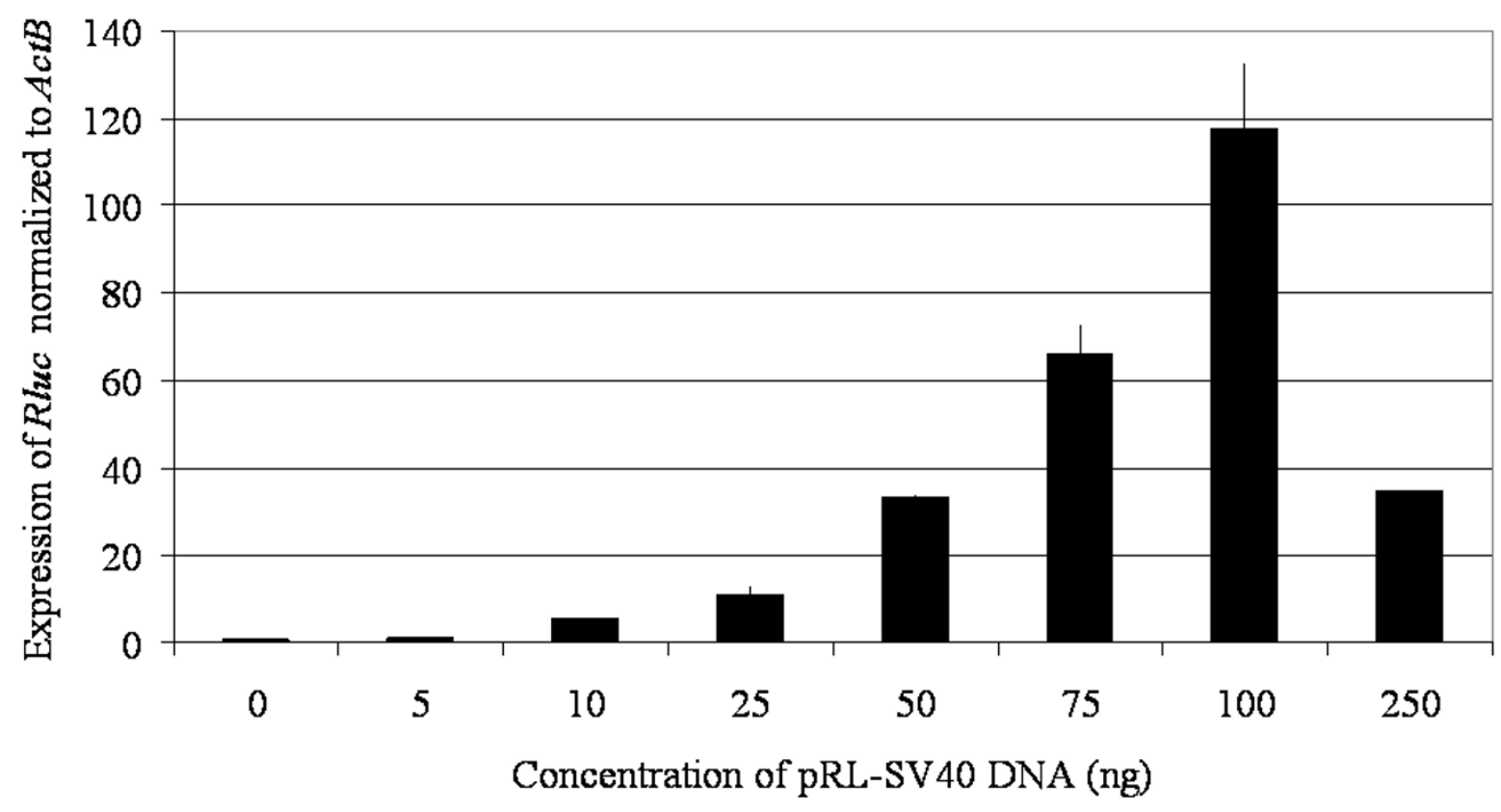

Figure 1 The effect of an increasing transfection concentration of pRL-SV40 DNA, a control plasmid encoding Rluc, on Rluc gene expression. Rluc expression was normalized to $A c t B$ gene expression. Each sample represents the expression of R/uc/ActB in a total RNA concentration of $0.2 \mu \mathrm{g}$. 
In $\mathrm{pMN86}$, a putative metal responsive element (MRE) is also present upstream of the $\mathrm{P}_{\mathrm{TK}}$ (Figure 2). Using the dual luciferase reporter assay system (Promega), we confirmed that the putative MRE conferred high cadmium (Cd) inducibility to the $\mathrm{P}_{\mathrm{TK}}$ in transiently transfected HEK293 cells (Table 1; pMN86 (MRE-P TK$-F l u c)$ vs. pMN85 ( $\mathrm{P}_{\mathrm{TK}}$-Fluc)).

In order to quantify the transcriptional activity of the induction of the $\mathrm{Cd}$ responsive MRE sequence using qPCR, we needed to find an appropriate normalization reference for these experiments. We planned to test the induction of cis-acting regulatory elements in the presence of a variety of compounds including metals (i.e. Cd), steroids (i.e. dexamethasone), cAMP activators (i.e. forskolin) and activators of protein kinase $C$ (i.e. phorbol-12-myristate 13-acetate (TPA)). Hence, the reference gene selected as a normalization control had to be stably expressed and unaffected by these inducers.

Traditionally, a chromosomal gene is selected as a reference gene for normalization. To this end, we tested a panel of 11 chromosomal genes as internal controls for
Table $1 \mathrm{Cd}$-dependent induction of MRE-directed expression of the firefly luciferase reporter enzyme activity

\begin{tabular}{|c|c|}
\hline & Normalized Fold Induction \\
\hline pMN85 ( $\mathrm{P}_{\mathrm{TK}}$-Fluc) & $1.00 \pm 0.00$ \\
\hline pMN86 (MRE-P TK -Fluc) & $212.77 \pm 8.92$ \\
\hline
\end{tabular}

The firefly and Renilla luciferase enzyme assays were conducted using the dual luciferase reporter assay system (Promega). The firefly luciferase enzyme activity was normalized to Renilla luciferase enzyme activity.

qPCR normalization using polymerase chain reaction (PCR) primers and fluorescent probes provided in the geNorm Housekeeping Selection Kit (PrimerDesign Ltd). The panel included the genes for $18 \mathrm{~S}$ rRNA (18S), betaactin $(A c t B)$, ATP synthase $(A t p 5 B)$, beta-2-microglobulin $(B 2 M)$, cytochrome c-1 (Cyc1), isoform 2 of the eukaryotic translation initiation factor 4A (Eif4A2), GAPDH, ribosomal protein L13a (RPL13A), subunit A of the succinate dehydrogenase complex (SDHA), DNA topoisomerase I (TOP1) and the zeta polypeptide of the tyrosine-3monooxygenase/tryptophan-5-monooxygenase activation



Figure 2 Schematic map of the plasmid pMN86. The plasmid encodes origins of replication (Col E1, Fl ori), a gene conferring resistance to the antibiotic ampicillin $(\mathrm{amp})$, the thymidine kinase promoter $\left(\mathrm{P}_{\mathrm{TK}}\right)$ upstream of the Firefly luciferase gene (Fluc) and five copies of the $\mathrm{Cd}$ responsive element (MRE). The MRE- $\mathrm{P}_{\text {TK }}$-Fluc cassette is flanked by two poly(A) signals to prevent transcriptional interference. 
protein (YWHAZ). The expression of these 11 chromosomal genes was measured in HEK293 cells that had been co-transfected with pMN85 or pMN86 and pRL-SV40 in the absence and presence of $\mathrm{Cd}$, dexamethasone, forskolin and TPA. The data was compared using three software applications; geNorm, Normfinder and BestKeeper (websites are shown in the Methods section).

GeNorm, a Visual Basic Application for Microsoft Excel, determines the expression stability of a potential reference gene by assigning each gene in a set of candidate reference genes an $M$ value [13]. This $M$ value is the mean pair-wise variation between any two genes in the data set being analyzed, one designated as the candidate gene and the other as the candidate reference gene. The gene with the highest $M$ value is then removed, and the stability measure of the remaining genes recalculated. As the calculation of $M$ value requires two genes, the calculation proceeds until the two best normalization reference genes, with the lowest $M$ value, remain in the analysis. As the expression profiles of the genes in the data set are compared to each other, the quality of the 'best' reference gene is dependent on the other genes that are selected for analysis, such that the geNorm ranking is reliable only if most of the genes selected as candidate reference genes are stably expressed and that the candidate reference genes are not co-regulated. To analyze our data with geNorm, the quantification cycle $(\mathrm{Cq})$ values generated in the $\mathrm{qPCR}$ experiments were converted to relative levels of expression using the method described by Livak and Schmittgen [23] and Vandesompele et al. [13], such that the lowest $\mathrm{Cq}$ value was set to 1 and all the other levels of expression for that gene were related to the lowest $\mathrm{Cq}$ value by the delta $\mathrm{Ct}$ method. These delta $\mathrm{Cq}$ values were then used to calculate the $\mathrm{M}$ values. geNorm analysis of the qPCR data for the 11 chromosomal reference genes (Table 2), indicated that the least suitable chromosomal reference gene was SDHA $(\mathrm{M}=2.77)$ and the most stable were $B 2 M$ and $Y W H A Z(M=0.10)$.

Normfinder is also a Visual Basic Application and it too assigns a stability value to the candidate reference genes. In contrast to geNorm, Normfinder uses a model-based approach to provide a value for the two most stable reference genes with the least intra- and inter-group variation [15]. The robustness of the Normfinder approach for the selection of reference genes for normalization was demonstrated by Andersen et al. [15]. As for the geNorm analysis, the experimental Cq values were converted to a linear scale of expression using the delta Ct method. This data loaded into Normfinder in two ways- (1) with the data separated into groups corresponding to treatment with the different activators, and (2) with all the treatment groups combined. In Normfinder, stability is expressed as a stability value in arbitrary units. Analysis of our data (Table 2) indicated that the RPL13A chromosomal gene was the most stable (stability value $=0.195 \pm 3.090)$ and that again $S D H A$ was the least stable gene (stability value $=31.894 \pm 10.087$ ).

BestKeeper is an Excel-based tool that calculates the variability in the expression of the pool of reference genes by analyzing the $\mathrm{Cq}$ values directly and defining variability by the standard deviation (SD) and coefficient of variance $(\mathrm{CV})$ [14]. All genes with SD values of greater than 1 are considered to be unsuitable reference genes. The gene with the lowest SD is considered to be the most suitable reference gene. BestKeeper then performs a comparative analysis based on the pair-wise correlations of the all the candidate reference genes to each other and generates a BestKeeper index. The comparison of this BestKeeper index to each candidate reference gene, using pair-wise correlation analyses, results in each candidate reference gene being assigned a value for the Pearson correlation coefficient ( $r$ ) and the probability (p). The most suitable reference gene is that with an $r$ value closest to 1.0, with the $\mathrm{p}$ value providing an indication of the significance of the $r$ value. The most unstable gene in the two previous analyses, SDHA, was not included in the BestKeeper analysis. The BestKeeper analysis (Table 2) of the potential reference genes highlighted B2M, Eif $4 A 2$ and RPL13A as being the most suitable normalization reference genes $(\mathrm{r}=0.974, \mathrm{p}=0.001)$ and TOP1 as the least suitable $(\mathrm{r}=0.704, \mathrm{p}=0.119)$.

When the results of the geNorm, Normfinder and BestKeeper analyses were summarized, the most stable reference genes were $B 2 M$ and RPL13A, as they were ranked as the most stable genes by two of the three software applications (Table 2). ActB is a chromosomal gene that is often used as a reference control. In fact, Suzuki et al. [12] report that $A c t B$ was one of the most popular reference controls, second only to GAPDH, being used in $32 \%$ of the cases examined. For these reasons, we selected $B 2 M$ and $A c t B$ as reference controls in further analyses. To compare Rluc on a transfected plasmid to the selected chromosomal reference genes $(B 2 M$ and $A c t B$ ) directly, the experimental Cq data for Rluc was incorporated into the geNorm, Normfinder and BestKeeper analysis (Table 3).

The stability values for Rluc were generally lower than for the chromosomal genes (0.63 (geNorm), 1.236 (Normfinder) and 0.694 (BestKeeper), as might be expected, since this reflects both the problem of variable transfection efficiency, and the lower levels of expression, meaning that more rounds of PCR are needed, accentuating any variable transfection efficiencies. However, the scores were still better than some, and close to the majority, of the generally used chromosomal genes, suggesting that this could still be considered as a 
Table 2 Ranking of chromosomal reference genes based on geNorm, Normfinder and BestKeeper analyses

\begin{tabular}{|c|c|c|c|c|c|c|c|c|}
\hline & \multicolumn{2}{|c|}{ geNorm } & \multicolumn{3}{|c|}{ Normfinder } & \multicolumn{3}{|c|}{ BestKeeper } \\
\hline & $\mathrm{M}$ value & Ranking & Stability value & Standard error & Ranking & {$[\mathrm{r}]$} & p-value & Ranking \\
\hline 185 & 0.13 & 3 & 1.425 & 1.540 & 8 & 0.958 & 0.003 & 6 \\
\hline$A c t B$ & 0.30 & 10 & 0.344 & 2.113 & 4 & 0.914 & 0.011 & 8 \\
\hline Atp5B & 0.22 & 7 & 0.255 & 2.539 & 3 & 0.973 & 0.001 & 4 \\
\hline$B 2 M$ & 0.10 & $1 / 2$ & 1.449 & 1.541 & 9 & 0.974 & 0.001 & $1 / 2 / 3$ \\
\hline $\mathrm{CyCl}$ & 0.16 & 4 & 1.126 & 1.543 & 6 & 0.933 & 0.007 & 7 \\
\hline Eif4A2 & 0.24 & 8 & 0.204 & 2.981 & 2 & 0.974 & 0.001 & $1 / 2 / 3$ \\
\hline GAPDH & 0.20 & 6 & 0.444 & 1.872 & 5 & 0.881 & 0.020 & 9 \\
\hline RPL13A & 0.19 & 5 & 0.195 & 3.090 & 1 & 0.974 & 0.001 & $1 / 2 / 3$ \\
\hline SDHA & 2.77 & 11 & 31.894 & 10.087 & 11 & nd & nd & nd \\
\hline TOP1 & 0.27 & 9 & 2.002 & 1.580 & 10 & 0.704 & 0.119 & 10 \\
\hline YWHAZ & 0.10 & $1 / 2$ & 1.225 & 1.539 & 7 & 0.959 & 0.002 & 5 \\
\hline
\end{tabular}

Highlighted regions represent the best ranked gene in the analysis. Abbreviations: nd- not determined; $M$ value- geNorm measure of expression stability, [r]BestKeeper coefficient of correlation, p-value- BestKeeper probability value.

candidate system for normalization [24-26]. According to the geNorm manual [http://medgen.ugent.be/ jvdesomp/genorm/geNorm_manual.pdf], genes with $M \geq 1.5$ are deemed unsuitable as reference genes. Based on this cut-off point, Rluc is not excluded as a suitable reference gene. Unfortunately, neither Andersen et al. [15] nor Pfaffl et al. [14] have reported guidelines on the range of acceptable stability values for the output of the Normfinder or BestKeeper applications, respectively $[14,15]$. This makes it difficult to determine the suitability of Rluc as a normalization control based on the values of the output of the Normfinder and BestKeeper applications. The output of all three bioinformatic analyses indicated that Rluc was not the most suitable gene for use as a reference control.

To evaluate the data from the geNorm, Normfinder and BestKeeper analyses for its biological validity, Rluc was compared to two chromosomal genes (B2M and $A c t B$ ) for its expression stability as a reference gene for normalization of a transfected plasmid expressing Fluc. qPCR was conducted on cDNA samples generated from HEK293 cells transfected with pMN85 $\left(\mathrm{P}_{\mathrm{TK}}-F l u c\right)$ or pMN86 (MRE-P TK $-F l u c$ ) and pRL-SV40 (Rluc). The Cq values obtained for the chromosomal internal controls $B 2 M$ and $A c t B$ were between 19 and 20 cycles, which were significantly lower than the Cq obtained for Rluc and for the target sequence, Fluc, which showed average Cq values of between 27 and 28 cycles (Table 4).

The Cq values were converted to number of copies with standard curves generated using known DNA concentrations of the respective linear DNA that encoded Fluc, Rluc, $B 2 M$ or $A c t B$ in $\mathrm{qPCR}$ reactions. The average numbers of copies of $B 2 M$ and $A c t B$ in the cDNA reactions in this experiment were 1500 -fold and 2700-fold higher than the average number of copies of Rluc (Figure 3). In contrast, the average number of copies of Fluc (under both control and induced conditions) was 30 fold higher than the average number of copies of Rluc. This highlighted the first problem associated with the selection of a chromosomal gene as an internal reference for the normalization of qPCR data from cells that had been transiently transfected with sequences of interest. The levels of expression of the chromosomal genes were much higher than the levels of the genes that were transiently transfected into cells. Problems associated with using highly expressed chromosomal genes as reference controls have been noted before, for example by Frost and Nilsen [27]. In addition, it has been noted that the use of reference and target genes with highly variable levels of expression would increase the noise within the assay system potentially resulting in a reduced sensitivity $[8,10]$. These dissimilar levels of

Table 3 Comparison of the ranking of B2M and ActB, the selected chromosomal reference genes, and Rluc, present on a transiently co-transfected plasmid, based on geNorm, Normfinder and BestKeeper analyses

\begin{tabular}{|c|c|c|c|c|c|c|c|c|}
\hline & \multicolumn{2}{|c|}{ geNorm } & \multicolumn{3}{|c|}{ Normfinder } & \multicolumn{3}{|c|}{ BestKeeper } \\
\hline & M value & Ranking & Stability value & Standard error & Ranking & {$[r]$} & $p$-value & Ranking \\
\hline$A c t B$ & 0.44 & $1 / 2$ & 1.342 & 0.515 & 3 & 0.931 & 0.001 & 2 \\
\hline$B 2 M$ & 0.44 & $1 / 2$ & 0.432 & 0.955 & 1 & 0.985 & 0.007 & 1 \\
\hline Rluc & 0.63 & 3 & 1.236 & 0.507 & 2 & 0.694 & 0.001 & 3 \\
\hline
\end{tabular}

Highlighted regions represent the best ranked gene in the analysis. Abbreviations: nd- not determined; $\mathrm{M}$ value- geNorm measure of expression stability, [r]BestKeeper coefficient of correlation, p-value- BestKeeper probability value. 
Table 4 Cq values obtained for Fluc, Rluc, B2M and ActB in $\mathrm{qPCR}$ reactions

\begin{tabular}{ccccc}
\hline & \multicolumn{4}{c}{ Gene } \\
& Fluc & Rluc & B2M & ActB \\
\hline Cq value \pm SD & $27.12 \pm 2.76$ & $27.98 \pm 0.44$ & $19.92 \pm 0.42$ & $19.08 \pm 0.52$
\end{tabular}

Abbreviations: Cq- quantification cycle, SD- standard deviation.

expression were made more apparent when the number of copies of Fluc was normalized to the internal reference genes as the ratios for normalized copies for Fluc/ $B 2 M$ and Fluc/ActB were very small compared to the ratio of Fluc/Rluc (Table 5).

The system was then tested for measuring changes in transcription levels. Following induction of the MREdirected transcription of Fluc in the presence of $\mathrm{Cd}$ and normalization against the internal chromosomal reference genes $B 2 M$ and $A c t B$, a 71-fold and 75-fold change in Fluc expression was calculated respectively (Table 6). In contrast, when Fluc was normalized to co-transfected Rluc, a lower 61-fold induction was calculated (Table 6). While the levels of fold induction of the Cd-responsive promoter-directed transcription of Fluc were not statistically different regardless of which gene was used as a normalization control, the levels of fold induction using the chromosomal genes as standards were consistently higher than when Rluc was used as the normalization control. More importantly, when the Fluc was normalized to B2M and $A c t B$, the error in the measurement, expressed as standard deviation, increased considerably to $23 \%$ and $35 \%$ of the total fold induction respectively compared to normalization to Rluc, where the standard deviation was $16 \%$ (Table 6). This is supported by the statistical analysis of the data which demonstrated that the calculated intervals for the fold induction and the standard deviation were smaller and less variable when Fluc was normalized to Rluc as opposed to $B 2 M$ or $A c t B$ (Table 7). This dramatic decrease in the standard deviation is most likely because, in addition to accounting for the quantity of RNA present in the starting material, the quality of the RNA and the efficiency of the enzymatic reactions during the experimental process, normalization of Fluc to Rluc also took into account the differences in the transfection efficiencies within the experiment.

\section{Conclusions}

A good control for normalization in qPCR experiments should be stably expressed at levels similar to those of the genes of interest, and should take into account all factors that could affect expression levels in the experiment, including transfection efficiency. While we observed that the chromosomal internal control genes $B 2 M$ and $A c t B$ were more stably expressed than transfected Rluc under the experimental conditions, the levels of expression of $B 2 M$ and $A c t B$ were much higher than those of genes transiently transfected into the cell.

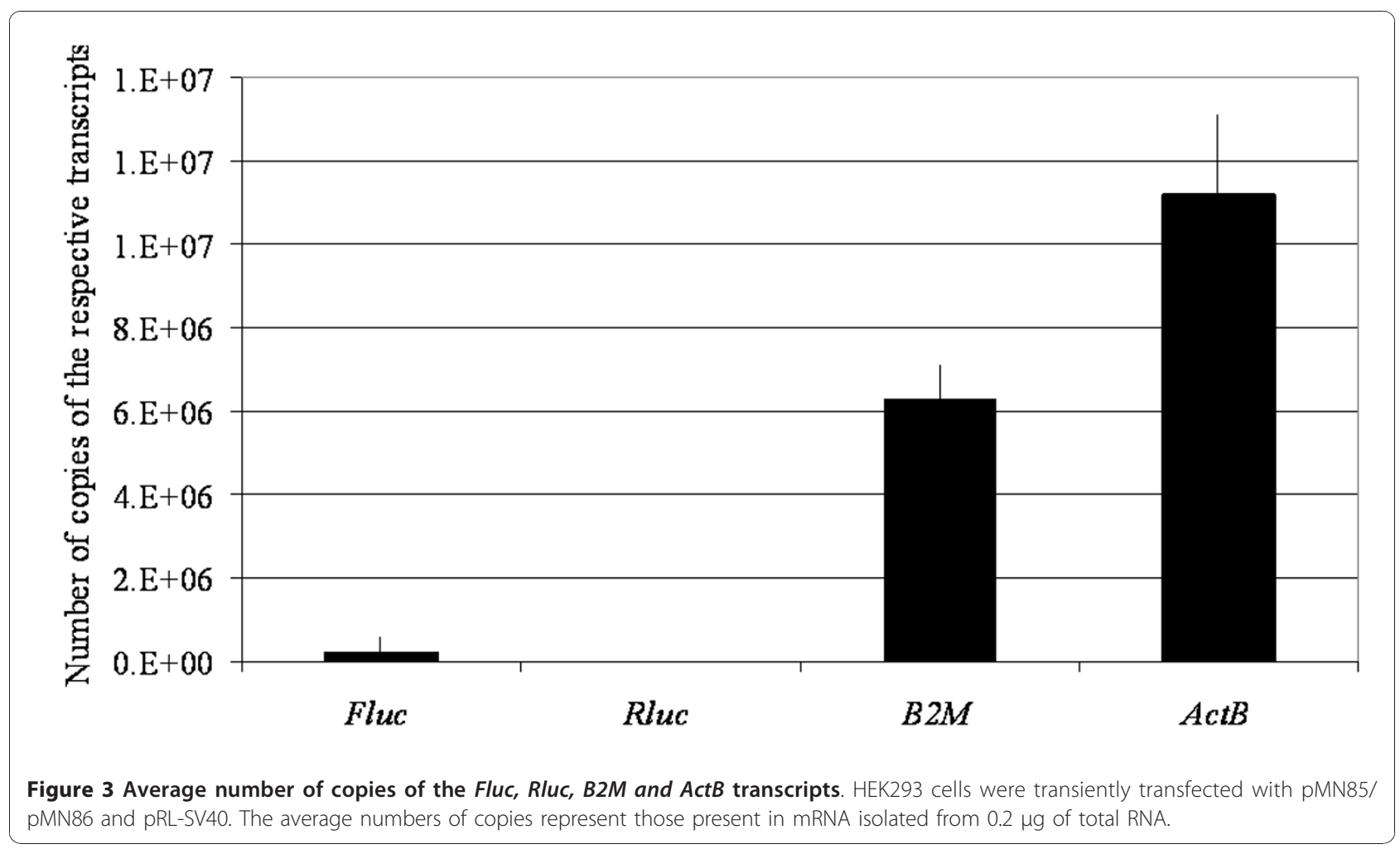


Table 5 Copies of Fluc normalized to the copies of the reference genes Rluc, B2M and ActB

\begin{tabular}{|c|c|c|c|c|c|c|c|}
\hline & & \multicolumn{2}{|l|}{ Fluc/Rluc } & \multicolumn{2}{|l|}{ Fluc/B2M } & \multicolumn{2}{|l|}{ Fluc/ActB } \\
\hline & & Normalized copies & \pm SD & Normalized copies $\left(\times 10^{-3}\right)$ & $\pm S D$ & Normalized copies $\left(\times 10^{-3}\right)$ & $\pm S D$ \\
\hline \multirow[t]{2}{*}{ pMN85 } & Untreated & 3.91 & 0.53 & 2.73 & 0.34 & 1.41 & 0.23 \\
\hline & $+\mathrm{Cd}$ & 5.11 & 1.00 & 2.85 & 0.46 & 1.86 & 0.25 \\
\hline \multirow[t]{2}{*}{ pMN86 } & Untreated & 3.50 & 0.51 & 2.22 & 0.43 & 1.22 & 0.31 \\
\hline & $+\mathrm{Cd}$ & 211.35 & 19.46 & 155.77 & 29.88 & 86.30 & 12.42 \\
\hline
\end{tabular}

The number of copies of the genes (Fluc/Rluc/B2M/ActB) was calculated using standard curves generated as described in the Methods. The normalized copy numbers for Fluc/B2M and Fluc/ActB were multiplied by a factor of $\left(1 \times 10^{3}\right)$ to allow the comparison of the ratio of Fluc normalized to the chromosomal reference genes $(B 2 M$ and $A c t B)$ to the ratio of Fluc normalized to the transiently co-transfected gene (Rluc). Abbreviations: SD- standard deviation.

The Rluc gene was expressed at levels similar to the gene of interest and was demonstrated by a number of biological analyses to be the most appropriate control, ensuring that differences in the transfection efficiencies between the samples were taken into account, thus significantly improving the reproducibility and the validity of the qPCR experiments.

\section{Methods}

\section{General Culture Conditions}

Escherichia coli DH5 $\alpha$ cells [28] were used for the construction, screening and propagation of plasmid constructs. E. coli DH5a cells were cultured in lysogeny broth (LB) or on LB agar containing $100 \mu \mathrm{g} / \mathrm{mL}$ ampicillin as described by Sezonov et al. [29]. HEK293 cells (LGC Standards, Middlesex, UK; ATCC Number CRL1573) [30] were maintained in Dulbecco's Modified Eagle Medium (DMEM, Invitrogen Ltd, Paisley, UK; 41966-029) supplemented with $4 \mathrm{mM}$ L-glutamine (Sigma-Aldrich, Dorset, UK; G7513) and 10\% foetal bovine serum (FBS; Biosera, East Sussex, UK; S1810/ $500)$ at $37^{\circ} \mathrm{C}$ in an atmosphere that contained $5 \% \mathrm{CO}_{2}$ as per the supplier's propagation instructions.

\section{Chromosomal reference genes}

The geNorm Housekeeping Gene Selection Kit (PrimerDesign Ltd, Southampton, UK; ge-PP-12-hu) was used as the panel of chromosomal reference genes. It included forward primers, Fluorescein (FAM)-labelled fluorescent probes and reverse primers for the chromosomal genes for

Table 6 Induction of Fluc expression in the presence of $\mathbf{C d}$

\begin{tabular}{|c|c|c|c|}
\hline & \multicolumn{3}{|c|}{ Fold induction } \\
\hline & $\begin{array}{c}\text { Fluc/Rluc } \pm \text { SD }(\% \\
\text { SD })\end{array}$ & $\begin{array}{c}\text { Fluc/B2M } \pm \text { SD }(\% \\
\text { SD })\end{array}$ & $\begin{array}{c}\text { Fluc/ActB } \pm \text { SD }(\% \\
\text { SD })\end{array}$ \\
\hline pMN85 & $1.34 \pm 0.42$ & $1.07 \pm 0.27$ & $1.35 \pm 0.28$ \\
\hline pMN86 & $61.42 \pm 10.12(16 \%)$ & $71.38 \pm 16.24(23 \%)$ & $74.92 \pm 25.80(35 \%)$ \\
\hline
\end{tabular}

18S, ActB, Atp5B, B2M, Cyc1, Eif4A2, GAPDH, RPL13A, SDHA, TOP1 and YWHAZ.

\section{Description of plasmids pMN85 and pMN86}

Plasmid pMN85 was derived from pGL3 Basic (Promega Corporation, Madison, WI, USA; E1751). DNA encoding (1) a multiple cloning cassette into which transcription factor binding sites could be inserted for analysis and (2) a $\mathrm{P}_{\mathrm{TK}}$ was inserted between the Kpn I and Hind III restriction enzyme sites upstream of Fluc in pGL3 generating pMN85. In pMN86 (Figure 2), five copies of a nucleotide sequence sensitive to induction in the presence of Cd (MRE; CGG GTG CGC CCG GCC CGA) were inserted between the Eco RI and Eco RV restriction enzyme sites upstream of $\mathrm{P}_{\mathrm{TK}}$.

\section{Transfection of HEK293 cells}

Plasmid DNA was prepared using the PureYield Plasmid Midiprep System (Promega Corporation, Madison, WI, USA; A2495). $1 \times 10^{6}$ HEK293 cells and, unless otherwise stated, a total of 100 ng DNA (50 ng pRLSV40 (Promega Corporation, Madison, WI, USA; E2231) and 50 ng of either pMN85 or pMN86) was used in each transfection. HEK293 cells were transfected using Genejuice (Novagen supplied by Merck Chemicals Ltd, Nottingham, UK; 70967-6) as recommended by the manufacturers instructions. Transfected HEK293 cells were incubated for 24 hours and subsequently induced with $12.5 \mu \mathrm{M} \mathrm{CdCl}{ }_{2}$ (Sigma-Aldrich, Dorset, UK; 439800), $2.5 \mu \mathrm{M}$ dexamethasone (Sigma-Aldrich, Dorset, UK; D8893), $25 \mu \mathrm{M}$ forskolin (Sigma-Aldrich, Dorset, UK; F6886) or $0.25 \mu \mathrm{M}$ TPA (Sigma-Aldrich,

Table 7 Credible Intervals for Fluc expression in the presence of $\mathbf{C d}$

\begin{tabular}{lccc}
\hline & \multicolumn{3}{c}{ 95\% Credible intervals for fold induction } \\
& Fluc/Rluc & Fluc/B2M & Fluc/ActB \\
\hline Mean fold induction & $(53.11,77.52)$ & $(58.92,106.48)$ & $(59.98,121.00)$ \\
Standard deviation & $(9.19,34.85)$ & $(16.30,74.62)$ & $(18.60,105.60)$ \\
\hline
\end{tabular}

The data were modelled using a lognormal distribution and uninformative priors. Random samples from the posterior were drawn and $95 \%$ credible intervals were calculated for both the mean fold induction and standard deviation. 
Dorset, UK; P1585) for 4 hours. Each experiment contained $\mathrm{n}=6$ biological replicates and experiments were repeated at least three times. Results between the experiments were comparable.

\section{Reporter enzyme assays}

The firefly and Renilla luciferase activities in $20 \mu \mathrm{L}$ HEK293 cell lysate were quantified with the dual luciferase reporter assay system as per the manufacturer's instructions (Promega Corporation, Madison, WI, USA; E1910) in a Luminoskan Ascent (Thermo Fisher Scientific, Waltham, Ma, USA) luminometer. The firefly and Renilla luciferase enzyme activities were measured for each biological sample. The firefly luciferase enzyme activity was normalized to the Renilla luciferase enzyme activity. The Cd-treated samples were then normalized to untreated samples yielding values for the fold induction of the firefly luciferase enzyme activity directed by the MRE in the presence of $\mathrm{Cd}$.

\section{RNA purification and CDNA synthesis}

Total RNA was prepared from $1 \times 10^{6}$ HEK293 cells using the miRNeasy mini kit (Qiagen Ltd, West Sussex, UK; 217004), mRNA was isolated from $4 \mu \mathrm{g}$ of total RNA with $25 \mu \mathrm{L}$ of the Dynabeads mRNA Purification Kit (Invitrogen Ltd, Paisley, UK; 610-06) and cDNA produced from the purified mRNA with $100 \mathrm{ng}$ random hexamers (Invitrogen Ltd, Paisley, UK; 48190011) or 500 $\mu \mathrm{g} / \mathrm{mL}$ oligo $\mathrm{dT}_{(12-18)}$ primer (Invitrogen Ltd, Paisley, UK; 18418-012), $2.5 \mathrm{mM}$ of each dNTP (Promega Corporation, Madison, WI, USA; U1240), 40 U RNaseOUT (Invitrogen Ltd, Paisley, UK; 10777-019), and $100 \mathrm{U}$ Superscript II enzyme (Invitrogen Ltd, Paisley, UK; 18064014) in a final volume of $20 \mu \mathrm{L}$. The cDNA synthesis reaction was incubated at $25^{\circ} \mathrm{C}$ for 10 minutes, $42^{\circ} \mathrm{C}$ for 60 minutes and finally, at $70^{\circ} \mathrm{C}$ for 15 minutes.

\section{qPCR conditions}

The cDNA $(20 \mu \mathrm{L})$ was diluted to $80 \mu \mathrm{L}$ with RNasefree $\mathrm{H}_{2} \mathrm{O}$ and $4 \mu \mathrm{L}$ was used in each qPCR reaction. For qPCR analysis of the chromosomal reference genes, in addition to $4 \mu \mathrm{L}$ of $\mathrm{cDNA}, 300 \mathrm{nM}$ proprietary primer mixture (PrimerDesign Ltd, Southampton, UK; ge-PP12-hu), which includes the forward primer, the FAMlabelled fluorescent probe and the reverse primer, and 1 $\times$ Lightcycler Probes Master (Roche Diagnostics Ltd, Burgess Hill, UK; 04887301001) was used. For the qPCR analysis of Fluc, Rluc, B2M and ActB gene expression, in addition to the cDNA, each reaction contained $2.4 \mu \mathrm{M}$ forward primer, $2.4 \mu \mathrm{M}$ reverse primer, $0.15 \mu \mathrm{M}$ fluorescent probe and $1 \times$ Lightcycler Probes Master. All primers were designed with Primer3Plus http://www. bioinformatics.nl/cgi-bin/primer3plus/primer3plus.cgi, synthesized by Eurofins MWG (Ebersberg, Germany) and the nucleotide sequence of the primers used in each reaction are shown in Table 8 . The cDNA was analyzed in a Lightcycler 480 (Roche Diagnostics Ltd, Burgess Hill, UK) using the following amplification conditions: (1) denaturation at $95^{\circ} \mathrm{C}$ for 10 minutes, (2) 50 cycles of amplification at $95^{\circ} \mathrm{C}$ for 15 seconds then $60^{\circ} \mathrm{C}$ for 30 seconds after which the level of fluorescence in the sample was measured and finally, (3) cooling to $40^{\circ} \mathrm{C}$ for 30 seconds.

\section{Analysis of the qPCR data for the reference genes}

Cq values obtained for HEK293 cells transiently transfected with pMN85/pMN86 and pRL-SV40 were grouped into 5 sets: (1) Untreated samples, $(2)+C d$, (3) + dexamethasone, (4) + forskolin and (5) + TPA. Each subset contained $n=6$ biological replicates. These sets of data were analyzed with three applications- geNorm http://medgen.ugent.be/ jvdesomp/genorm/, Normfinder http://www.mdl.dk/publicationsnormfinder. htm and BestKeeper http://www.gene-quantification. com/bestkeeper.html\#download as described by the documentation for the software applications.

\section{Generation of standard curves for Fluc, Rluc, B2M and ActB}

Linear dsDNA was used to generate standard curves for qPCR, after Hou et al. [31] highlighted the benefits of using linear DNA in preference to circular DNA. The Fluc, Rluc, B2M and ActB PCR products generated in the qPCR reactions above were cloned into the pGEM-T-Easy plasmid using the pGEM-T-Easy Vector System I (Promega Corporation, Madison, WI, USA; A1360). Ligations were transformed into Subcloning Efficiency $^{\mathrm{Tm}}$ DH5 $\alpha^{\mathrm{Th}}$ Competent Cells (Invitrogen Ltd, Paisley, UK; 18265-017). Plasmid constructs were screened for the desired insert using the GoTaq ${ }^{\circledR}$ Flexi DNA Polymerase (Promega Corporation, Madison, WI, USA; M8291) in PCR reactions that contained $2 \mathrm{mM}$ $\mathrm{MgCl}_{2}$, $25 \mathrm{ng}$ DNA, $0.2 \mathrm{mM}$ of each dNTP (Promega Corporation, Madison, WI, USA; U1240), $1 \mu \mathrm{M}$ gene specific forward primer (Table 8), $1 \mu \mathrm{M}$ gene specific reverse primer (Table 8) and 0.5 U GoTaq DNA polymerase in a final volume of $25 \mu \mathrm{L}$. The PCR conditions were: (1) $92^{\circ} \mathrm{C}$ for 2 minutes, 1 cycle; (2) $92^{\circ} \mathrm{C}$ for 30 seconds, $52^{\circ} \mathrm{C}$ for 30 seconds, $72^{\circ} \mathrm{C}$ for 1 minute, 30 cycles and $(3) 72^{\circ} \mathrm{C}$ for 2 minutes, 1 cycle. The DNA in the PCR reactions was separated on a $1 \%$ Seakem LE Agarose gel (Lonza Biologics plc, Slough, Berkshire, UK; 50004) containing 0.01\% Sybr Safe DNA gel stain (Invitrogen Ltd, Paisley, UK; S33102). Constructs that showed a positive PCR reaction were selected for validation by DNA sequencing.

The PCR fragment of interest (Fluc/Rluc/B2M/ActB) was amplified from the pGEM-T-Easy plasmid 
Table 8 Nucleotide sequences of the primers used in the qPCR reactions and the length of the amplicons generated

\begin{tabular}{lll}
\hline Transcript (amplicon length) & Primer & Sequence $\mathbf{( 5}^{\prime}$ to $\mathbf{3}^{\prime}$ ) \\
\hline Fluc (137 nts) & prMJ296 & AGGTGGCTCCCGCTGAT \\
& prMJ297 & FAM-CGGGAAGACCTGCGACACCTGCGT-BHQ1 \\
& prMJ298 & CATCGTCTTCCGTGCTCCA \\
\hline RluC (140 nts) & prMJ274 & GCAGAAGTTGGTGTGAGG \\
& prMJ272 & HEX-CTCACTATAGGCTAGCCACCATGACTTCGAAAG-BHQ1 \\
& prMJ276 & TCATCCGTTCCTTGTTCTG \\
\hline B2M (125 nts) & prMJ348 & TCTCTGCTCCCCACCTCTAA \\
& prMJ349 & FAM-CCAGCCCTCCTAGAGCTACC-BHQ1 \\
& prMJ350 & ATCTGAGCAGGTGCTCCAC \\
\hline ActB (142 nts) & prMJ351 & CTCGGCCACATTGTGAACTT \\
& prMJ352 & FAM-ATGCTCGCTCCAACCGAC-BHQ1 \\
\hline
\end{tabular}

Abbreviations: BHQ1- Black Hole Quencher 1, FAM- Fluorescein, HEX- hexachlorofluorescein phosphoramidite.

construct encoding the DNA of interest using the reagents and reaction conditions shown for the $\mathrm{qPCR}$ reactions. The PCR products were purified from a $1 \%$ Seakem LE Agarose gel (Lonza Biologics plc, Slough, Berkshire, UK; 50004) containing 0.01\% Sybr Safe DNA gel stain (Invitrogen Ltd, Paisley, UK; S33102) using the Zymoclean ${ }^{\text {tx }}$ Gel DNA Recovery Kit (Zymo Research Corporation, Orange, CA, USA; D4007) and quantified on a Nanodrop spectrophotometer (Thermo Fisher Scientific, Waltham, Ma, USA). The DNA copy number was calculated based on the formulas reported by Whelan et al. [32].

Weight in Daltons $(\mathrm{g} / \mathrm{mol})=(\mathrm{bp}$ size of ds product $)(330 \mathrm{Da} \times 2 \mathrm{nt} / \mathrm{bp})$

Copy number $=$ Weight in Daltons $(\mathrm{g} / \mathrm{mol}) /$ Avogadro's number $\left(6.022 \times 10^{23} 1 / \mathrm{mol}\right)$

where bp- base pairs, ds- double stranded and ntnucleotides. With the DNA concentration and the copy number, an accurate number of molecules could be calculated. The DNA was diluted from $1 \times 10^{9}$ copies/uL to 0 copies/uL in a 10 -fold dilution series. $\mathrm{qPCR}$ reactions were conducted on the DNA dilutions using the same reagents and reaction conditions reported for the qPCR reactions above. A standard curve was generated by plotting the $\mathrm{Cq}$ value against the $\log$ of the copy number. The unknown samples were compared to the standard curve and the copy number of the unknown targets calculated.

$$
\mathrm{E}=10^{(-1 / \text { slope of the curve })}
$$

where the optimal efficiency of the qPCR reaction was 2. Primer pairs that have qPCR efficiencies of between 1.6 and 2.4 are typically used [33]. The efficiency of the qPCR reactions and errors were calculated with the Lightcycler 480 qPCR software (Roche Diagnostics Ltd, Burgess Hill, UK) and are shown in Table 9.

\section{qPCR data analysis}

Cq values obtained for HEK293 cells transiently transfected with pMN85/pMN86 and pRL-SV40 were grouped into 4 sets: (1) pMN85 - Untreated, (2) pMN85 - + Cd, (3) pMN86 - Untreated, (4) pMN86 $-+\mathrm{Cd}$. The $\mathrm{Cq}$ values were converted to copy numbers using the Fluc, Rluc, B2M and ActB standard curves. The copy number of Fluc was normalized to the copy number of Rluc, B2M or $A c t B$. The copy number of the normalized $\mathrm{Cd}$-treated samples were then compared to the copy number of the normalized untreated samples for pMN85 and pMN86 yielding values for the fold induction of the Fluc directed by the MRE in the presence of $\mathrm{Cd}$.

\section{Statistical analysis of qPCR data}

The data were analysed using JAGS [34], a Gibbs sampler for hierarchical models and Coda [35], a tool for examining Markov Chain Monte Carlo runs. The data were modelled using a log-normal distribution, with uninformative normal priors for the mean parameter and uninformative gamma priors for the precision. For each set of data, 10 independent runs were used, each with a burn in of 20000 iterations and 100000 samples taken. From these samples, 95\% credible intervals were calculated for the posterior mean and standard deviation of the fold induction (Table 7).

Table 9 The efficiencies and errors of the qPCR standard curves as calculated by the Lightcycler $\mathbf{4 8 0}$ software (Roche Diagnostics Ltd, Burgess Hill, UK)

\begin{tabular}{lll}
\hline Standard curve & Efficiency & Error \\
\hline FluC & 1.878 & 0.001 \\
RluC & 1.878 & 0.001 \\
B2M & 2.027 & 0.053 \\
ACtB & 1.994 & 0.002 \\
\hline
\end{tabular}




\section{List of Abbreviations}

18S: $18 \mathrm{~S}$ rRNA gene; ActB: beta actin gene; Atp5B: ATP synthase gene; amp: $\beta$-lactamase gene conferring resistance to ampicillin; B2M: beta-2microglobulin gene; BHQ1: Black Hole Quencher 1; bp: base pairs; Cd: Cadmium; CMV: cytomegalovirus; Cq: quantification cycle; Ct: threshold cycle; CV: coefficient of variance; DMEM: Dulbecco's Modified Eagle Medium; ds: double stranded; E: efficiency of the GPCR reaction; Eif4A2: eukaryotic translation initiation factor $4 \mathrm{~A}$, isoform 2 gene;FAM:Fluorescein;FBS:foetal bovine serum; Fluc: firefly luciferase gene; GAPDH: glyceraldehyde-3phosphate dehydrogenase gene; HEK293: Human Embryonic Kidney 293; HEX: hexachlorofluorescein phosphoramidite; HMBS: hydroxymethylbilane synthase gene; HSV: herpes simplex virus; LB: lysogeny broth; M value: geNorm measure of expression stability; MRE: metal responsive element;nt: nucleotides; ori: origin of replication;p-value:probability; PCR: polymerase chain reaction; $\mathrm{P}_{\mathrm{TK}}$ : thymidine kinase promoter; $\mathrm{Qpcr}$ : quantitative real-time polymerase chain reaction; r: BestKeeper coefficient of correlation; Rluc: Renilla luciferase gene; RPL13A: ribosomal protein L13a gene; SD: standard deviation; SDHA: succinate dehydrogenase complex, subunit A gene; SV40: simian virus 40; TK: thymidine kinase; TOP1: DNA topoisomerase I gene; TPA: phorbol-12-myristate 13-acetate; YWHAZ: tyrosine-3-monooxygenase/ tryptophan-5-monooxygenase activation protein (zeta polypeptide) gene.

\section{Acknowledgements}

We would like to thank Sarah Cumming for discussions during the preparation of this manuscript and the EPSRC for funding this study through a Basic Technology Grant (EP/E0327545/1).

\section{Author details}

${ }^{1}$ Institute of Molecular, Cell and Systems Biology, College of Medical, Veterinary and Life Sciences, University of Glasgow, Glasgow, G12 8QQ, UK. ${ }^{2}$ Inference Group, School of Computing Science, University of Glasgow, Glasgow, G12 8QQ, UK. ${ }^{3}$ Systems Biology Ireland and the Conway Institute, University College Dublin, Belfield, Dublin 4, Ireland.

\section{Authors' contributions}

$\mathrm{MJ}, \mathrm{KP}, \mathrm{PM}$ and JY conducted the experiments. RD conducted the statistical analyses on the experimental data. MJ, WK and ARP drafted the manuscript. WK and ARP designed the project and secured funding for the study. All authors read and approved the final text.

Received: 3 August 2010 Accepted: 31 December 2010 Published: 31 December 2010

\section{References}

1. Ginzinger DG: Gene quantification using real-time quantitative PCR: an emerging technology hits the mainstream. Experimental Hematology 2002, 30:503-512.

2. Higuchi R, Fockler $C$, Dollinger $G$, Watson $R$ : Kinetic $P C R$ analysis: real-time monitoring of DNA amplification reactions. Biotechnology 1993, 11:1026-1030

3. Heid CA, Stevens J, Lival KJ, Williams PM: Real time quantitative PCR. Genome Research 1996, 6:986-994.

4. Nolan T, Hands RE, Bustin SA: Quantification of mRNA using real-time RTPCR. Nature Protocols 2006, 1:1559-1582.

5. Bustin SA: Quantification of mRNA using real-time reverse transcription PCR (RT-PCR): trends and problems. Journal of Molecular Endocrinology 2002, 29:23-39.

6. Bustin SA: Absolute quantification of mRNA using real-time reverse transcription polymerase chain reaction. Journal of Molecular Endocrinology 2000, 25:169-193.

7. Aerts JL, Gonzalesm MI, Topalian SL: Selection of appropriate reference genes to assess expression of tumor antigens using real-time PCR. Biotechniques 2004, 36:84-91.

8. Dheda K, Huggett JF, Bustin SA, Johnson MA, Rook G, Zumla A: Validation of housekeeping genes for normalizing RNA expression in real-time PCR. Biotechniques 2004, 37:112-119.

9. Radonic A, Thulke S, Mackay IM, Landt O, Siegert W, Nitsche A: Guideline to reference gene selection for quantitative real-time PCR. Biochemical and Biophysical Research Communications 2004, 313:856-862.

10. Dheda K, Huggett JF, Chang JS, Kim LU, Bustin SA, Johnson MA, Rook GAW, Zumla A: The implications of using an inappropriate reference gene for real-time reverse transcription PCR data normalization. Analytical Biochemistry 2005, 344:141-143.

11. Huggett J, Dheda K, Bustin S, Zumla A: Real-time RT-PCR normalization; strategies and considerations. Genes and Immunity 2005, 6:279-284.

12. Suzuki T, Higgins PJ, Crawford DR: Control selection for RNA quantitation. Biotechniques 2000, 29:332-337.

13. Vandesompele J, De Preter K, Pattyn F, Poppe B, Van Roy N, De Paepe A, Speleman F: Accurate normalization of real-time quantitative RT-PCR data by geometric averaging of multiple internal reference genes. Genome Biology 2002, 3(7):research0034.1-0034.11.

14. Pfaffl MW, Tichopad A, Prgomet C, Neuvians TP: Determination of stable housekeeping genes, differentially regulated target genes and sample integrity: BestKeeper - Excel-based tool using pair-wise correlations. Biotechnology Letters 2004, 26:509-515.

15. Andersen $C L$, Jensen $J L$, Orntoft TF: Normalization of real-time quantitative reverse transcription PCR data: a model based variance estimation approach to identify genes sioted for normalization, applied to bladder and colon cancer data sets. Cancer Research 2004, 64:5245-5250.

16. pRL Renilla Luciferase Reporter Vectors. Technical Bulletin No. TB550 Promega Corporation, Madison WI USA 2008.

17. Bruening W, Giasson B, Mushynski W, Durham HD: Activation of stressactivated MAP protein kinases up-regulates expression of transgenes driven by the cytomegalovirus immediate/early promoter. Nucleic Acids Research 1998, 26:486-489.

18. Svensson RU, Barnes JM, Rokhlin OW, Cohen MB, Henry MD: Chemotherapeutic Agents Up-regulate the Cytomegalovirus Promoter: Implications for Bioluminescence Imaging of Tumor Response to Therapy. Cancer Res 2007, 67:10445-10454.

19. Thavathiru E, Das GM: Activation of pRL-TK by 12S E1A oncoprotein: drawbacks of using an internal reference reporter in transcription assays. Biotechniques 2001, 31:528-532.

20. Matuszyk J, Ziolo E, Cebrat M, Kockel I, Strzadala L: Nurr1 affects pRL-TK but not phRG-B internal control plasmid in genetic reporter system. Biochemical and Biophysical Research Communications 2002, 294:1036-1039

21. Ho CKM, Strauss JF III: Activation of the control reporter plasmids pRL-TK and PRL-SV40 by multiple GATA transcription factors can lead to aberrant normalization of transfection efficiency. BMC Biotechnology 2004, 4:10-14.

22. Yin W, Xiang P, Li Q: Investigations of the effectof DNA size in transient transfection assay using dual luciferase system. Analytical Biochemistry 2005, 346:289-294

23. Livak KJ, Schmittgen TD: Analysis of relative gene expression data using real-time quantitative PCR and the $2^{-\Delta \Delta C t}$ method. Methods 2001, 25:402-408.

24. Perez R, Tupac-Yupanqui I, Dunner S: Evaluation of suitable reference genes for gene expression studies in bovine muscular tissue. BMC Molecular Biology 2008, 9:79.

25. Valente V, Teixeira SA, Neder L, Okamoto OK, Oba-Shinjo SM, Marie SKN, Scrideli CA, Paco-Larson ML, Carlotti CG Jr: Selection of suitable housekeeping genes for expression analysis in glioblastoma using quantitative RT-PCR. BMC Molecular Biology 2009, 10:17.

26. Lallemant B, Evrard A, Combescure C, Chapuis H, Chambon G, Raynal C, Reynaud C, Sabra O, Joubert D, Hollande F, Lallemant JG, Lumbroso S, Brouillet JP: Reference gene selection for head and neck squamous cell carcinoma gene expression studies. BMC Molecular Biology 2009, 10:78.

27. Frost $P$, Nilsen $F$ : Validation of reference genes for transcription profiling in the salmon louse Lepeophtheirus salmonis by quantitative real-time PCR. Vet Parasitology 2003, 118:169-174.

28. Hanahan D: Studies on transformation of Escherichia coli with plasmids. Journal of Molecular Biology 1983, 166:557-580.

29. Sezonov G, Joseleau-Petit D, D'Ari R: Escherichia coli physiology in LuriaBertani broth. Journal of Bacteriology 2007, 189:8746-8749.

30. Graham FL, Smiley J, Russell WC, Nairn R: Characteristics of a human cell line transformed by DNA from human adenovirus Type 5. Journal of General Virology 1977, 36:59-72.

31. Hou Y, Zhang H, Miranda L, Lin S: Serious overestimation in quantitative PCR by circular (supercoiled) plasmid standard: microalgal pcna as the model gene. PLOS ONE 2010, 5:e9545.

32. Whelan JA, Russell NB, Whelan MA: A method for using absolute quantification of CDNA using real-time PCR. Journal of Immunological Methods 2003, 278:261-269. 
33. Yun JJ, Heisler LE, Hwang IIL, Wilkins O, Lau SK, Hyrcza M, Jayabalasingham B, Jin J, McLaurin J, Tsao MS, Der SD: Genomic DNA functions as a universal external standard in quantitative real-time PCR. Nucleic Acids Research 2006, 34:e85.

34. Plummer M: JAGS: A program for analysis of Bayesian graphical models using Gibbs sampling. Proceedings of the 3rd International Workshop on Distributed Statistical Computing; 2003.

35. Plummer M, Best N, Cowles K, Vines K: CODA: Convergence Diagnosis and Output Analysis for MCMC. $R$ News 2006, 6:11-17.

doi:10.1186/1471-2199-11-103

Cite this article as: Jiwaji et al.: The Renilla luciferase gene as a reference gene for normalization of gene expression in transiently transfected cells. BMC Molecular Biology 2010 11:103.

Submit your next manuscript to BioMed Central and take full advantage of:

- Convenient online submission

- Thorough peer review

- No space constraints or color figure charges

- Immediate publication on acceptance

- Inclusion in PubMed, CAS, Scopus and Google Scholar

- Research which is freely available for redistribution

Submit your manuscript at www.biomedcentral.com/submit
C Biomed Central 